\title{
DFT STUDY OF THE TUBULAR SIZE EFFECTS ON THE PROPERTIES OF ZIGZAG BORON NITRIDE NANOTUBES
}

\section{FATEMEH SHEKHOLIA LAVASANI ${ }^{a}$, ABOLGHASEM SHAMELI ${ }^{\text {b* }}$, EBRAHIM BALALI ${ }^{a}$}

\begin{abstract}
Density functional theory (DFT) studies were performed on representative zigzag models of boron nitride nanotubes (BNNTs) with different structural sizes. To achieve minimized-energy structures and their properties, first, optimization calculations were performed at the B3LYP/6$311 G^{*}$ theoretical level for all models. Subsequently, density of states (DOS) plots, nuclear magnetic resonance (NMR) spectroscopy chemical shielding, natural bonding orbital (NBO) and nuclear quadrupole resonance (NQR) spectroscopy parameters were calculated. The results indicated that the values of energy gap, polarity and electrical charge detect the effects of structural growth in diameter and length.
\end{abstract}

Keywords: Boron nitride; Nanotube; Density functional theory; Tubular size

\section{INTRODUCTION}

Boron nitride nanotubes (BNNTs) are among those nanostructures which are structurally analogues to carbon nanotubes (CNTs) but they show different properties such as distinct electronic behavior [1-4]. Contrary to CNTs, BNNTs are always wide gap semiconductors with the energy gap (Eg) in the range of 4.5 to $4.9 \mathrm{eV}$, independently of tubular chirality, diameter and number of walls. In addition, BNNTs also have high degree of radial flexibility and high Young modulus, excellent mechanical properties, high thermal conductivity, and high oxidation resistance [5-8].

a Department of Chemistry, Pharmaceutical Sciences Branch, Islamic Azad University, Tehran, Iran.

${ }^{b}$ Department of Chemistry, Faculty of Science, Omidiyeh Branch, Islamic Azad University, Omidiyeh, Iran.

*Corresponding author: Shameli678@gmail.com 
In this work, properties of $(n, 0)$ zigzag BNNTs $(n=4-7)$ with various lengths were investigated based on density functional theory (DFT) calculations. To this aim, the optimized geometries, density of states (DOS), nuclear magnetic resonance (NMR) chemical shielding, natural bonding orbital (NBO) and nuclear quadrupole resonance (NQR) parameters were calculated at the B3LYP/6-311G* level of DFT using the Gaussian 03 package.

\section{RESULTS AND DISCUSSION}

First, the model structures of $(4,0),(5,0),(6,0)$ and $(7,0)$ BNNTs with various tubular lengths were optimized to obtain minimized-energy structures. Based on the optimizations, bond lengths, bond angles, energies, dipole moments, the highest occupied and the lowest unoccupied molecular orbitals (HOMO and LUMO) were evaluated (Tables 1-3).

Table 1.Optimized bond length of BNNTs $(4,0),(5,0),(6,0),(7,0)$

\begin{tabular}{cccccccc}
\hline $\begin{array}{c}\text { BNNTS } \\
(4,0)\end{array}$ & $\begin{array}{c}\text { Bond } \\
\text { Length } \\
\left(\mathrm{A}^{0}\right)\end{array}$ & $\begin{array}{c}\text { BNNTS } \\
(5,0)\end{array}$ & $\begin{array}{c}\text { Bond } \\
\text { Length } \\
\left(\mathrm{A}^{0}\right)\end{array}$ & $\begin{array}{c}\text { BNNTS } \\
(6,0)\end{array}$ & $\begin{array}{c}\text { Bond } \\
\text { Length } \\
\left(\mathrm{A}^{0}\right)\end{array}$ & $\begin{array}{c}\text { BNNTS } \\
(7,0)\end{array}$ & $\begin{array}{c}\text { Bond } \\
\text { Length } \\
\left(\mathrm{A}^{0}\right)\end{array}$ \\
\hline B1N2 & 1.48 & B1N2 & 1.47 & B1H43 & 1.19 & B1H57 & 1.19 \\
B1N3 & 1.48 & B1N3 & 1.47 & B1N63 & 1.46 & B1N100 & 1.46 \\
B1H41 & 1.18 & B1H61 & 1.18 & B1N75 & 1.46 & B1N109 & 1.46 \\
N2B4 & 1.44 & N2B6 & 1.45 & B2N51 & 1.46 & B2N108 & 1.46 \\
N2B15 & 1.48 & N2B19 & 1.47 & B2N69 & 1.45 & B2N109 & 1.45 \\
N3B5 & 1.44 & N3B4 & 1.45 & B2N70 & 1.46 & B2N117 & 1.46 \\
N3B7 & 1.48 & N3B7 & 1.47 & B3N70 & 1.46 & B3N99 & 1.46 \\
B4N6 & 1.49 & B4N5 & 1.47 & B3N75 & 1.45 & B3N100 & 1.45 \\
B4N16 & 1.49 & B4N10 & 1.47 & B3N76 & 1.46 & B3N108 & 1.46 \\
B5N6 & 1.49 & N5B6 & 1.47 & B4H44 & 1.19 & B4H58 & 1.19 \\
B5N10 & 1.49 & N5B26 & 1.44 & B4N75 & 1.46 & B4N91 & 1.46 \\
N6B23 & 1.44 & B6N20 & 1.47 & B4N81 & 1.46 & B4N100 & 1.46 \\
B7N8 & 1.48 & B7N8 & 1.47 & B5N76 & 1.46 & B5N86 & 1.46 \\
B7H42 & 1.18 & B7H62 & 1.18 & B5N81 & 1.45 & B5N91 & 1.45 \\
N8B9 & 1.44 & N8B9 & 1.45 & B5N82 & 1.46 & B5N99 & 1.46 \\
N8B11 & 1.48 & N8B11 & 1.47 & B6H45 & 1.19 & B6H59 & 1.19 \\
B9N10 & 1.49 & B9N10 & 1.47 & B6N81 & 1.46 & B6N85 & 1.46 \\
B9N14 & 1.49 & B9N14 & 1.47 & B6N87 & 1.46 & B6N91 & 1.46 \\
N10B21 & 1.44 & N10B25 & 1.44 & B7N82 & 1.46 & B7N77 & 1.46 \\
B11N12 & 1.48 & B11N12 & 1.47 & B7N87 & 1.45 & B7N85 & 1.45 \\
B11H43 & 1.18 & B11H63 & 1.18 & B7N89 & 1.46 & B7N86 & 1.46 \\
N40H48 & 1.02 & N60H70 & 1.02 & N59H60 & 1.01 & N95H96 & 1.01 \\
\hline
\end{tabular}


Bond lengths are in the ranges of $1.44-1.48 \AA$ for $\mathrm{B}$ and $\mathrm{N}$ atoms and in the ranges of $1.01-1.02 \AA$ for $\mathrm{N}$ and $\mathrm{H}$ atoms. Increasing the size of BNNTs, in terms of diameters and lengths, decrease the bond length of B-N and $\mathrm{N}-\mathrm{H}$. The $\mathrm{B}-\mathrm{H}$ bond lengths are between 1.18-1.19 $\AA$. Decreasing the size of BNNTs results in decreasing the $\mathrm{B}-\mathrm{H}$ bond length.

Table 2 shows that the $\mathrm{B}-\mathrm{N}-\mathrm{B}$ and $\mathrm{H}-\mathrm{N}-\mathrm{B}$ bond angles are in the range of $107.5^{\circ}-119.5^{\circ}$ and $108.9^{\circ}-117.6^{\circ}$, respectively, in which the numbers are increased to near maximum by increasing the size of nanotube. In addition, the bond angle for $\mathrm{H}-\mathrm{B}-\mathrm{N}$ is $121.1^{\circ}-122.4^{\circ}$ approaching $122.4^{\circ}$ by decreasing the size of BNNTs ring. Dipole moments for nanotubes are recorded between 1.68-7.3 Debye. Except BNNTs (4,0), the values are increased by increasing the diameter of the nanotube ring. The energy of the BNNTs was measured in a range of $-43.5 \mathrm{eV}$ to $-121.7 \mathrm{eV}$, in which the stability of the BNNTs are reasonably increased by the size of nanotubes.

Table 2. Optimized bond angles of BNNTs $(4,0),(5,0),(6,0),(7,0)$

\begin{tabular}{cccccccc}
\hline $\begin{array}{c}\text { BNNTS } \\
(4,0)\end{array}$ & $\begin{array}{c}\text { Bond } \\
\text { Angle }\end{array}$ & $\begin{array}{c}\text { BNNTS } \\
(5,0)\end{array}$ & $\begin{array}{c}\text { Bond } \\
\text { Angle }\end{array}$ & $\begin{array}{c}\text { BNNTS } \\
(6,0)\end{array}$ & $\begin{array}{c}\text { Bond } \\
\text { Angle }\end{array}$ & BNNTS (7,0) & $\begin{array}{c}\text { Bond } \\
\text { Angle }\end{array}$ \\
\hline N2B1N3 & 112.0 & N2B1N3 & 114.3 & B1N69B2 & 118.7 & H57B1N100 & 121.1 \\
N2B1H41 & 122.4 & N2B1H61 & 121.7 & B1N69B12 & 114.1 & H57B1N109 & 121.1 \\
N3B1H41 & 122.4 & N3B1H61 & 121.7 & B2N69B12 & 118.6 & B3N100B4 & 119.0 \\
B1N2B4 & 116.2 & B1N2B6 & 117.9 & B2N70B3 & 111.5 & B20N101B21 & 114.3 \\
B1N2B15 & 107.5 & B1N2B19 & 111.3 & B2N70B18 & 118.7 & B20N101B23 & 119.0 \\
B4N2B15 & 116.1 & B6N2B19 & 117.9 & B3N70B18 & 118.7 & B21N101B23 & 119.0 \\
B1N3B5 & 116.1 & B1N3B4 & 117.9 & B19N71B20 & 112.4 & B34N102B35 & 114.3 \\
B1N3B7 & 107.5 & B1N3B7 & 111.4 & B19N71B30 & 118.5 & B34N102B37 & 118.9 \\
B5N3B7 & 116.2 & B4N3B7 & 117.9 & B20N71B30 & 118.5 & B35N102B37 & 118.9 \\
N2B4N6 & 119.3 & N3B4N5 & 119.7 & B31N72B32 & 112.3 & B48N103B49 & 114.2 \\
N2B4N16 & 119.3 & N3B4N10 & 119.7 & B31N72B42 & 118.7 & B48N103B51 & 119.1 \\
N6B4N16 & 113.9 & N5B4N10 & 115.8 & B32N72B42 & 118.7 & B49N103B51 & 119.1 \\
N3B5N6 & 119.3 & B4N5B6 & 108.4 & B29N73B30 & 112.3 & B50N104B51 & 115.8 \\
N3B5N10 & 119.3 & B4N5B26 & 118.0 & B29N73B32 & 118.4 & B50N104H105 & 117.6 \\
N6B5N10 & 113.9 & B6N5B26 & 118.0 & B30N73B32 & 118.4 & B51N104H105 & 117.6 \\
B4N6B5 & 103.3 & N2B6N5 & 119.8 & B17N74B18 & 112.4 & B36N106B37 & 114.3 \\
B4N6B23 & 116.4 & N2B6N20 & 119.7 & B17N74B20 & 118.5 & B36N106B49 & 118.9 \\
B5N6B23 & 116.4 & N5B6N20 & 115.8 & B18N74B20 & 118.5 & B37N106B49 & 118.9 \\
N3B7N8 & 112.0 & N3B7N8 & 114.2 & B1N75B3 & 118.6 & B22N107B23 & 114.3 \\
N3B7H42 & 122.4 & N3B7H62 & 121.7 & B1N75B4 & 114.1 & B22N107B35 & 118.9 \\
N8B7H42 & 122.4 & N8B7H62 & 121.7 & B3N75B4 & 118.6 & B23N107B35 & 119.0 \\
B7N8B9 & 116.1 & B7N8B9 & 117.9 & B3N76B5 & 111.6 & B2N108B3 & 113.5 \\
B7N8B11 & 107.5 & B7N8B11 & 111.4 & B3N76B17 & 118.8 & B2N108B21 & 119.2 \\
\hline
\end{tabular}


FATEMEH SHEKHOLIA LAVASANI, ABOLGHASEM SHAMELI, EBRAHIM BALALI

\begin{tabular}{cccccccc}
\hline $\begin{array}{c}\text { BNNTS } \\
(4,0)\end{array}$ & $\begin{array}{c}\text { Bond } \\
\text { Angle }\end{array}$ & $\begin{array}{c}\text { BNNTS } \\
(5,0)\end{array}$ & $\begin{array}{c}\text { Bond } \\
\text { Angle }\end{array}$ & $\begin{array}{c}\text { BNNTS } \\
(6,0)\end{array}$ & $\begin{array}{c}\text { Bond } \\
\text { Angle }\end{array}$ & BNNTS $(7,0)$ & $\begin{array}{c}\text { Bond } \\
\text { Angle }\end{array}$ \\
\hline B9N8B11 & 116.2 & B9N8B11 & 117.9 & B5N76B17 & 118.8 & B3N108B21 & 119.2 \\
N8B9N10 & 119.3 & N8B9N10 & 119.7 & B20N77B21 & 112.4 & B1N109B2 & 119.0 \\
N8B9N14 & 119.3 & N8B9N14 & 119.7 & B20N77B29 & 118.5 & B1N109B14 & 115.9 \\
N2B1N3 & 112.0 & N2B1N3 & 114.3 & B1N69B2 & 118.7 & B1N100B3 & 119.0 \\
N2B1H41 & 122.4 & N2B1H61 & 121.7 & B1N69B12 & 114.1 & B1N100B4 & 115.9 \\
N3B1H41 & 122.4 & N3B1H61 & 121.7 & B2N69B12 & 118.6 & B3N100B4 & 119.0 \\
B1N2B4 & 116.2 & B1N2B6 & 117.9 & B2N70B3 & 111.5 & B20N101B21 & 114.3 \\
B1N2B15 & 107.5 & B1N2B19 & 111.3 & B2N70B18 & 118.7 & B20N101B23 & 119.0 \\
B4N2B15 & 116.1 & B6N2B19 & 117.9 & B3N70B18 & 118.7 & B21N101B23 & 119.0 \\
B1N3B5 & 116.1 & B1N3B4 & 117.9 & B19N71B20 & 112.4 & B34N102B35 & 114.3 \\
B1N3B7 & 107.5 & B1N3B7 & 111.4 & B19N71B30 & 118.5 & B34N102B37 & 118.9 \\
B5N3B7 & 116.2 & B4N3B7 & 117.9 & B20N71B30 & 118.5 & B35N102B37 & 118.9 \\
N2B4N6 & 119.3 & B51N60H7 & 114.1 & B31N72B32 & 112.3 & B48N103B49 & 114.2 \\
N2B4N16 & 119.3 & B55N60H7 & 114.1 & B37N55H56 & 117.2 & B48N103B51 & 119.1 \\
B37N39H47 & 108.9 & N5B4N10 & 115.8 & B32N72B42 & 118.7 & B53N80H81 & 117.6 \\
N3B5N6 & 119.3 & B4N5B6 & 108.4 & B29N73B30 & 112.3 & B50N104B51 & 115.8 \\
\hline
\end{tabular}

Table 3. Optimized physical properties of BNNTs $(4,0),(5,0),(6,0),(7,0)$

\begin{tabular}{cccccc}
\hline BNNTs & $\begin{array}{c}\mathrm{E} \\
(\mathrm{e} . \mathrm{v})\end{array}$ & $\begin{array}{c}\text { Dipole Moment } \\
\text { (debye) }\end{array}$ & $\begin{array}{c}\text { HOMO } \\
(\mathrm{e} . \mathrm{v})\end{array}$ & $\begin{array}{c}\text { LUMO } \\
(\mathrm{e} . \mathrm{v})\end{array}$ & $\begin{array}{c}\text { Gap } \\
(\mathrm{e} . \mathrm{v})\end{array}$ \\
\hline$(7,0)$ & -121701.905 & 7.3010 & -6.51836248 & -1.78265812 & -4.73570436 \\
$(6,0)$ & -91293.87084 & 5.4374 & -6.55319384 & -2.2613172 & -4.29187664 \\
$(5,0)$ & -65224.56566 & 1.6823 & -6.41441264 & -2.9769928 & -3.43741984 \\
$(4,0)$ & -43492.37267 & 6.5626 & -6.63727892 & -4.07200368 & -2.56527524 \\
\hline
\end{tabular}

\section{HOMO and LUMO parameters and density of states (DOS)}

Electronic density of states (DOS) of individual BNNTs are shown in Fig.1. HOMO and LUMO parts are distinguished by the orbital distribution patterns.

The electronic properties of BNNTs are often characterized in terms of their HOMO and LUMO energies and the corresponding energy gap ( $\mathrm{Eg}$ in $\mathrm{eV}$ ). The distribution patterns of the frontier molecular orbitals (HOMO and LUMO) [9] is shown in Fig. 1 and Table 3. Based on Fig. 1, the variance between HOMO and LUMO in $(4,0)$ BNNT is $-2.56 \mathrm{eV}$, which is less than BNNT $(6,0)$ and $(7,0)$. The ordering of energy gap in the four models were: $\operatorname{Eg}(4,0)<\operatorname{Eg}(5,0)<\operatorname{Eg}(6,0)$ $<$ Eg $(7,0)$. Based on these results, we concluded that the electrical conductivity was increased by decreasing the size of nanotube. 
DFT STUDY OF THE TUBULAR SIZE EFFECTS ON THE PROPERTIES OF ZIGZAG BORON ...
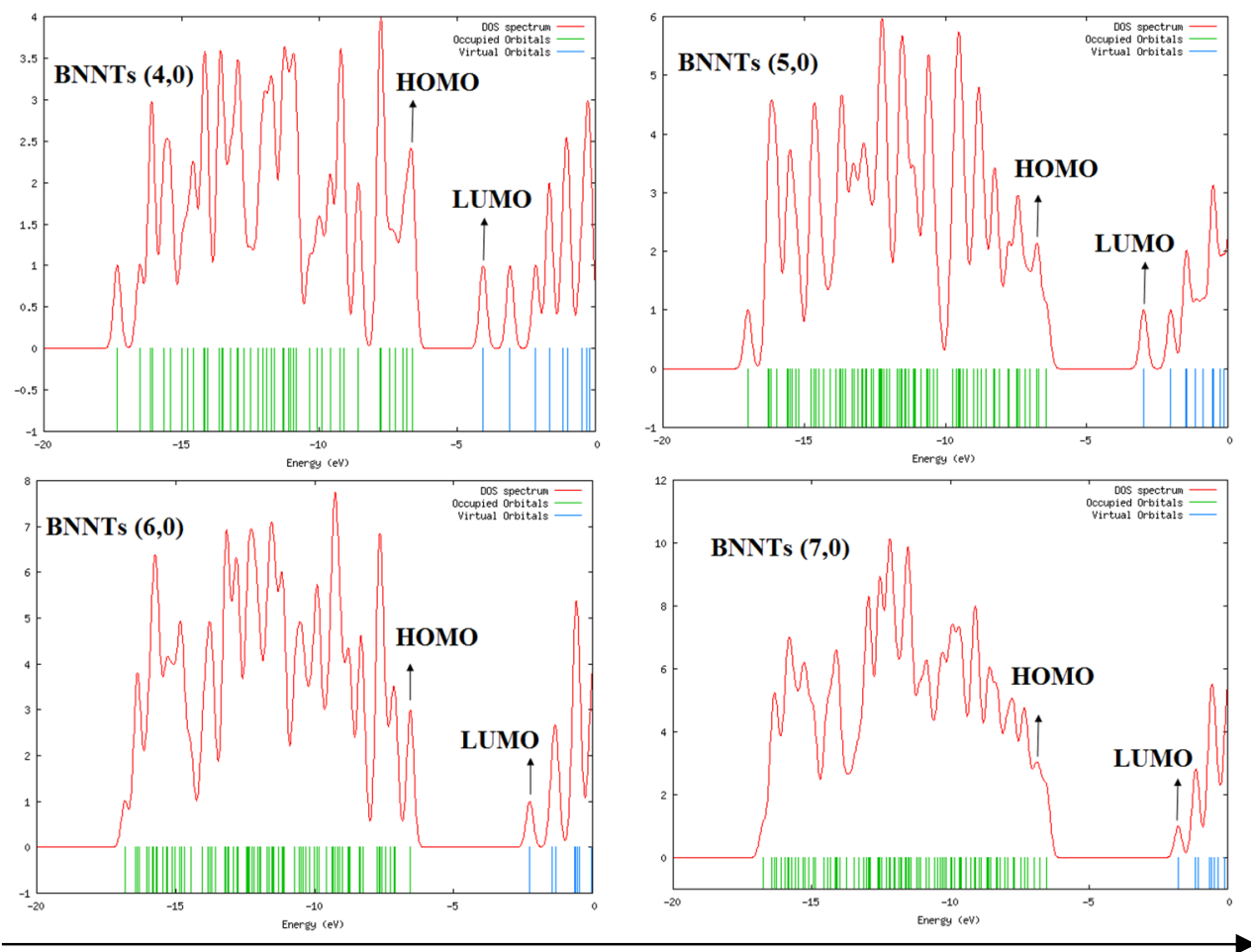

Energy (ev)

Figure 1. Diagram of the DOS per energy for BNNT;

$(4,0),(5,0),(6,0)$ and $(7,0)$ models

\section{Natural bonding orbital (NBO)}

For a deeper understanding of the interaction between adsorbent surface and the adsorbate, the partial electronic charge densities were calculated using NBO analysis [9, 10], which opens a better view for discussing the atomic charge distributions (Table 4). In all these cases by expanding the size of the nanotube, the electronic population of the models increase and the maximum can be found in BNNTs $(7,0)$.

Table 4. The NBO information for BNNT $(4,0),(5,0),(6,0)$ and $(7,0)$

\begin{tabular}{ccrccr}
\hline BNNTs & Charge & Core & Valence & Rydberg & Total \\
\hline 7,0 & 0.00000 & 223.87871 & 461.24112 & 0.88017 & 686.00000 \\
6,0 & 0.00000 & 167.90644 & 347.36740 & 0.72615 & 516.00000 \\
5,0 & 0.00000 & 119.92988 & 249.45157 & 0.61855 & 370.00000 \\
4,0 & 0.00000 & 79.94934 & 167.48747 & 0.56319 & 248.00000 \\
\hline
\end{tabular}




\section{NUCLEAR MAGNETIC RESONANCE}

Chemical shielding (CS) parameters in NMR were evaluated for the optimized BNNTs. To calculate the CS tensors, the gauge included atomic orbital (GIAO) approach was used [11]. The calculated CS tensors in principal axes system (PAS) $\left(\sigma_{33}>\sigma_{22}>\sigma_{11}\right)$ were converted to measurable CS NMR parameters, isotropic and anisotropic CS ( $\mathrm{C}_{\mathrm{SI}}$ and $\left.\mathrm{C}_{\mathrm{SA}}\right)$ using Eqs. (1) and (2) [12, 13]. The evaluated NMR parameters are listed in Table 5.

$$
\begin{aligned}
& \mathrm{C}_{\mathrm{SI}}=\left(\sigma_{11}+\sigma_{22}+\sigma_{33}\right) / 3 \\
& \mathrm{C}_{\mathrm{SA}}=\sigma_{33}-\left(\sigma_{22}+\sigma_{11}\right) / 2
\end{aligned}
$$

The results show that the $C_{S I}$ values of optimized $(4,0),(5,0),(6,0)$ and $(7,0)$ BNNTs for the B-H bonds are around 26 and 27 ppm while for the $\mathrm{N}-\mathrm{H}$ bonds are around 29 and $30 \mathrm{ppm}$. The isotropic and anisotropic chemical shielding $\left(\mathrm{C}_{S I}\right.$ and $\left.\mathrm{C}_{S A}\right)$ parameters were calculated for the ${ }^{11} \mathrm{~B},{ }^{1} \mathrm{H}$ and ${ }^{15} \mathrm{~N}$ atoms present in the pristine structures. In addition, the tensors were converted to the isotropic $\mathrm{C}_{\mathrm{S}}\left(\mathrm{C}_{\mathrm{SI}}\right)$ and the anisotropic $\mathrm{C}_{\mathrm{S}}\left(\mathrm{C}_{\mathrm{SA}}\right)$ parameters. The $C_{S I}$ is the average value of the eigenvalues of the $C_{S}$ tensors, (Eq.1), and the orientation of the eigenvalues of the $\mathrm{C}_{\mathrm{S}}$ tensors into the $z$-axis plays a dominant role in determining the value of the $C_{S A}$ parameter, (Eq.2) [14]. These results show that due to the anisotropic effect, the hydrogen of $\mathrm{B}-\mathrm{H}$ is de-shielding while hydrogen of $\mathrm{N}-\mathrm{H}$ is shielding.

Table 5 shows that the anisotropic values of $B$ atoms are between 42-60 ppm, which could imply de-shielding effects and tendency to the weaker magnetic fields.

\begin{tabular}{|c|c|c|c|c|c|c|c|c|c|}
\hline \multirow[b]{2}{*}{ atom } & \multicolumn{4}{|c|}{ BNNTs 7,0} & \multicolumn{5}{|c|}{ BNNTs 6,0} \\
\hline & CSA & $\begin{array}{l}\text { Aniso- } \\
\text { tropy }\end{array}$ & CSI & $\begin{array}{l}\text { Isotro } \\
\text {-pic }\end{array}$ & atom & CSA & $\begin{array}{l}\text { Anisot- } \\
\text { ropy }\end{array}$ & CSI & $\begin{array}{c}\text { Isotropi } \\
\text { C }\end{array}$ \\
\hline B3 & 30.3 & 43.5 & 74.9 & 74.9 & B2 & 11.5 & 46.2 & 73.1 & 73.1 \\
\hline B5 & 31.0 & 43.5 & 74.9 & 74.9 & B7 & 11.5 & 46.1 & 73.0 & 73.0 \\
\hline B10 & 41.0 & 59.5 & 67.8 & 67.8 & B19 & 8.4 & 44.7 & 71.8 & 71.8 \\
\hline B12 & 42.0 & 59.6 & 67.7 & 67.7 & B22 & 8.4 & 44.7 & 71.8 & 71.8 \\
\hline B16 & 28.9 & 42.3 & 73.4 & 73.4 & B31 & 9.4 & 44.9 & 72.4 & 72.4 \\
\hline N103 & 133.0 & 192.1 & 124.7 & 124.7 & N90 & 165.7 & 196.8 & 113.4 & 113.4 \\
\hline N124 & 138.3 & 196.4 & 122.0 & 122.0 & N91 & 171.3 & 195.5 & 114.4 & 114.4 \\
\hline $\mathrm{N} 125$ & 138.3 & 196.8 & 121.3 & 121.3 & N92 & 163.8 & 194.9 & 115.3 & 115.3 \\
\hline N126 & 143.8 & 202.3 & 121.1 & 121.1 & N93 & 166.1 & 191.7 & 118.2 & 118.2 \\
\hline
\end{tabular}

Table 5. NMR parameter for BNNTs $(4,0),(5,0),(6,0),(7,0)$ 
DFT STUDY OF THE TUBULAR SIZE EFFECTS ON THE PROPERTIES OF ZIGZAG BORON ...

\begin{tabular}{|c|c|c|c|c|c|c|c|c|c|}
\hline \multirow[b]{2}{*}{ atom } & \multicolumn{4}{|c|}{ BNNTs 5,0} & \multirow[b]{2}{*}{ atom } & \multicolumn{4}{|c|}{ BNNTs 4,0} \\
\hline & CSA & $\begin{array}{l}\text { Aniso- } \\
\text { tropy }\end{array}$ & $\mathrm{CSI}$ & $\begin{array}{l}\text { Isotro } \\
\text { pic }\end{array}$ & & CSA & $\begin{array}{l}\text { Aniso- } \\
\text { tropy }\end{array}$ & CSI & $\begin{array}{c}\text { Isotro- } \\
\text { pic }\end{array}$ \\
\hline B21 & 37.9 & 49.4 & 68.1 & 68.1 & B30 & 15.5 & 60.5 & 64.0 & 64.0 \\
\hline B52 & 43.5 & 50.5 & 72.3 & 72.3 & B26 & 15.5 & 60.5 & 64.0 & 64.0 \\
\hline B54 & 43.4 & 50.5 & 72.3 & 72.3 & B13 & 17.3 & 61.4 & 63.6 & 63.6 \\
\hline B55 & 15.3 & 54.5 & 67.6 & 67.6 & B5 & 17.3 & 61.4 & 63.6 & 63.6 \\
\hline N36 & 157.9 & 193.3 & 102.6 & 102.6 & N32 & 130.9 & 134.9 & 73.3 & 73.3 \\
\hline N56 & 90.5 & 126.0 & 126.5 & 126.5 & N10 & 183.0 & 193.5 & 77.8 & 77.8 \\
\hline N59 & 90.1 & 126.0 & 126.5 & 126.5 & N3 & 37.1 & 242.5 & 25.1 & 25.1 \\
\hline N27 & 155.6 & 192.6 & 102.6 & 102.6 & N16 & 183.0 & 193.5 & 77.8 & 77.8 \\
\hline
\end{tabular}

However, the values of $\mathrm{N}$ atoms are between 126-250 ppm, which could reveal the shielding effects and tendency to higher magnetic fields.

In other words, $\mathrm{N}$ and $\mathrm{B}$ atoms show positive and negative anisotropic behaviors, respectively. That's why the $\mathrm{B}$ and $\mathrm{N}$ atoms were observed in weaker and stronger magnetic fields, respectively.

\section{NUCLEAR QUADRUPOLE RESONANCE}

Nuclear quadrupole resonance (NQR) spectroscopy is among the most important techniques to characterize the composition of chemical structures. In contrast to NMR, the NQR analysis could be found in the absence of magnetic field in nuclear conversion as a zero-filed technique. The NQR resonance is a connection between electric field gradient (EFG) and Nuclear quadrupole resonance in which the charge distribution occurred. However, the EFG shows the location of nuclei in material in which the linked valance electrons of the atoms are modified. NQR frequency shows the absolute conversion of an element. This frequency in a composite or a crystal is proportional to nuclear quadrupole resonance, nuclei properties and EFG of neighbor nuclei.

The relation between results and the calculations could be investigated using EFG tensors with the main axis of the system. The calculated EFG tensors were converted to quadrupole coupling constants $\left(\mathrm{C}_{\mathrm{Q}}\right)$ and asymmetry parameters $\left(\eta_{\mathrm{Q}}\right)$, which are directly measured by nuclear quadrupole resonance (NQR) spectroscopy [15].

Using Eqs.(3) and (4), the $\mathrm{C}_{Q}$ and $\eta_{Q}$ parameters could be found if $\left|q_{z z}\right|<\left|q_{y y}\right|<\left|q_{x x}\right|$. The standard quantity of the nuclear quadrupole momentum $\mathrm{Q}$ [16] are listed in Table 6.

$$
\begin{aligned}
& C_{Q}=e^{2} Q_{z z} h^{-1} \\
& \eta_{Q}=\left|\left(q_{x x}-q_{y y}\right) / q_{z z}\right|
\end{aligned}
$$


FATEMEH SHEKHOLIA LAVASANI, ABOLGHASEM SHAMELI, EBRAHIM BALALI

Table 6. NQR parameter for BNNTs $(4,0),(5,0),(6,0),(7,0)$

\begin{tabular}{|c|c|c|c|c|c|c|c|c|c|c|c|}
\hline \multirow[t]{2}{*}{ atom } & \multicolumn{2}{|c|}{ BNNTs 4,0 } & \multirow[t]{2}{*}{ atom } & \multicolumn{2}{|c|}{ BNNTs 5,0} & \multirow[t]{2}{*}{ atom } & \multicolumn{2}{|c|}{ BNNTs 6,0} & \multirow[t]{2}{*}{ atom } & \multicolumn{2}{|c|}{ BNNTs 7,0} \\
\hline & $\eta \mathrm{Q}$ & $\mathrm{C}_{\mathrm{Q}}$ & & $\eta \mathrm{Q}$ & $\mathrm{CQ}$ & & $\eta \mathrm{Q}$ & $\mathrm{CQ}_{\mathrm{Q}}$ & & $\eta \mathrm{Q}$ & $\mathrm{C}_{\mathrm{Q}}$ \\
\hline B5 & 0.00 & 2.78 & B1 & 0.00 & 2.78 & B1 & 0.00 & 2.78 & B1 & 0.00 & 2.78 \\
\hline B7 & 0.00 & 2.78 & B4 & 0.00 & 2.78 & B2 & 0.00 & 2.78 & B3 & 0.00 & 2.78 \\
\hline B13 & 0.00 & 2.78 & B13 & 0.00 & 2.78 & B3 & 0.00 & 2.78 & B4 & 0.00 & 2.78 \\
\hline B15 & 0.00 & 2.78 & B15 & 0.00 & 2.78 & B8 & 0.00 & 2.78 & B8 & 0.00 & 2.78 \\
\hline B17 & 0.00 & 2.78 & B26 & 0.00 & 2.78 & B9 & 0.00 & 2.78 & B9 & 0.00 & 2.78 \\
\hline B21 & 0.00 & 2.78 & B34 & 0.00 & 2.78 & B15 & 0.00 & 2.78 & B10 & 0.00 & 2.78 \\
\hline B26 & 0.00 & 2.78 & B42 & 0.00 & 2.78 & B18 & 0.00 & 2.78 & B11 & 0.00 & 2.78 \\
\hline B30 & 0.00 & 2.78 & B51 & 0.00 & 2.78 & B20 & 0.00 & 2.78 & B17 & 0.00 & 2.78 \\
\hline B35 & 0.00 & 8.03 & B52 & 0.00 & 2.78 & B23 & 0.00 & 2.78 & B18 & 0.00 & 2.78 \\
\hline B39 & 0.00 & 8.03 & B54 & 0.00 & 2.78 & B27 & 0.00 & 2.78 & B20 & 0.00 & 2.78 \\
\hline average & 0.00 & 3.19 & & 0.00 & 2.78 & & 0.00 & 2.78 & & 0.00 & 2.78 \\
\hline
\end{tabular}

Table 6 shows that, the values of $\eta_{Q}$ for BNNTs are near zero while for $\mathrm{C}_{\mathrm{Q}}$ average 2.78. In addition, the quadrupole momentum is mostly constant and aligned in z-axis.

\section{CONCLUSIONS}

In this work, the properties of different boron nitride nanotubes with zigzag chirality were investigated. The results show that by increasing the size, in terms of diameters and lengths of the BNNTs, the energy gap and polarity increased and the hybridisation form becomes SP2. In NMR of the BNNTS, the nitrogen atoms is shielding to the higher magnetic field and boron atoms de-shielding to the lower magnetic field. However, in NBO spectroscopy, by increasing the size of the nanotube, the electrical charge increased. In addition, based on the NQR, the effect of the gradient of the electrical field on nuclear quadrupole momentum for different size of the BNNTs were mostly equal and no obvious changes were observed.

\section{COMPUTATIONAL DETAIL}

In this study, the structure of Boron Nitride nanotube (BNNT) $(4,0)$ (20 B, $20 \mathrm{~N}$ and $8 \mathrm{H}$ atoms), BNNT $(5,0)(30 \mathrm{~B}, 30 \mathrm{~N}$ and $10 \mathrm{H}$ atoms), BNNT $(6,0)(42 \mathrm{~B}, 42 \mathrm{~N}$ and $12 \mathrm{H}$ atoms) and BNNT $(7,0)(56 \mathrm{~B}, 56 \mathrm{~N}$ and $14 \mathrm{H}$ atoms) were investigated. All atomic geometries of the Boron Nitride nanotubes were firstly optimized at the B3LYP (exchange-correlation functional and the $6-311 \mathrm{G}^{*}$ level standard basis set) to reach the minimum 
energy structures with the optimized values of bond lengths and angles. Figure 2 shows the graphical representation of the optimized geometry of BNNT $(7,0)$, drawn using Hyperchem and GaussView 5.0 software. Note that the sizes of nanotubes were increased based on the proportionality of diameter to length, in which one ring systems was added to increase either diameter or length of nanotubes.
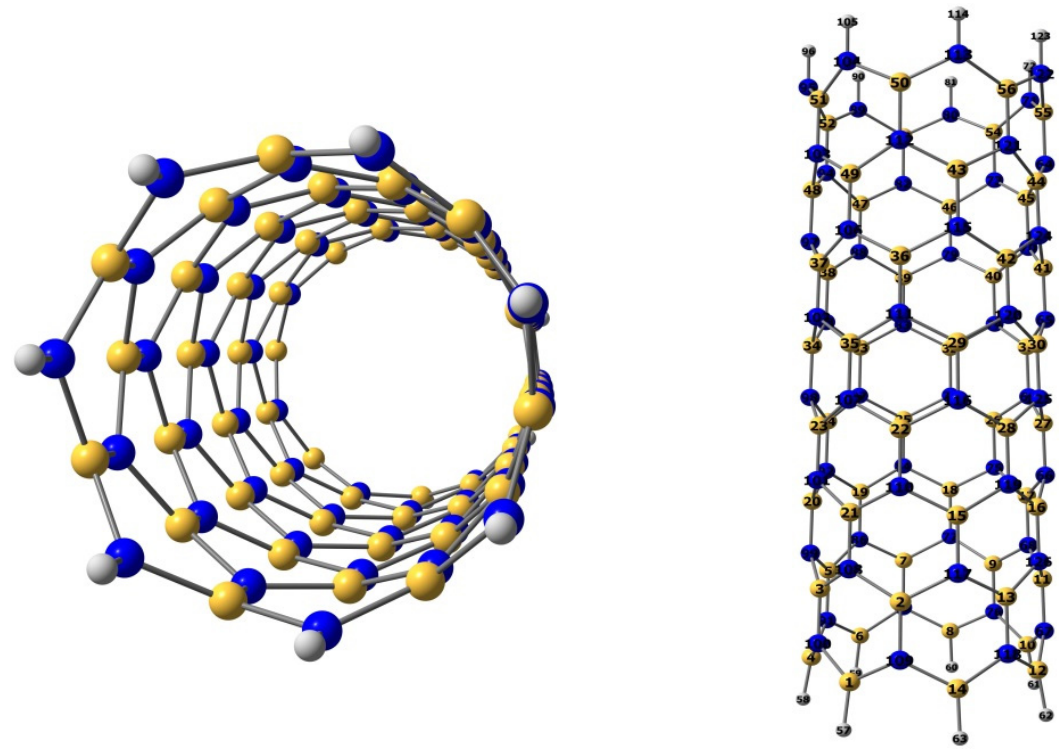

Figure 2. The optimized structure of BNNT $(7,0)$

Afterwards, the parameters of total energy, energy gap, dipole moment, nuclear quadrupole resonance (NQR), nuclear magnetic resonance spectroscopy (NMR) and Natural bond orbital analysis (NBO) were obtained for the optimized structures by performing single point energy calculations at the computational level of B3LYP. All calculations were performed using the Gaussian 03 package. The results were summarized in Tables 1-6.

\section{ACKNOWLEDGMENTS}

The author is grateful to Pharmaceutical Sciences Branch, Islamic Azad University of Tehran for the support of this work. 


\section{REFERENCES}

1. D. Golberg, Y. Bando, K. Kurashima, T. Sato, Chemical Physics Letters, 2000, 323,185.

2. D. Golberg, M. Mitome, Y. Bando, C.C. Tang and C.Y. Zhi, Applied Physics A, 2009, 88, 347.

3. Y. Yap, "B-C-N Nanotubes and Related Nanostructures", Springer, New York 2009.

4. P. Ayala, A. Rubio and T. Pichler, Reviews in Modern Physics, 2010, 82, 1843.

5. R. Arenal, X. Blase, A. Loiseau, Advanced Physics, 2010, 59, 101.

6. G. Chopra, A. Zettl, Solid State Communications, 1998, 105, 297.

7. Y.H. Kim, K.J. Chang and S.G. Louie, Physical Review B, 2001, 63, 205408.

8. A. Freitas, S. Azevedo, J.R. Kaschny, Physica E, 2016, 84, 444.

9. J. Kaur, P. Singla, N. Goel, Applied Surface Science, 2014, 328, 632.

10. R. Ditchfield, W.J. Hehre, J.A. Pople, Journal Chemical Physics. 1971, 54, 724.

11. M. Mirzaei, Journal of Molecular Modeling. 2010, 17, 89.

12. A. Shameli, E. Balali, R. Khadivei, S. Shojaei, Oriental Journal of Chemistry, 2016, 32(1), 291.

13. V.A. Ferreira, H.W. Leite Alves, Journal Crystal Growth, 2008, 310, 3973.

14. M. Rezaei-Sameti, Arabian Journal of Chemistry, 2011, 8, 168.

15. M. Mirzaei, M. Giahi, Physica E, 2010, 42, 1667.

16. P. Pyykko, Molecular Physics, 2001, 99, 1617. 